\title{
ACM-BCS Visions of Computer Science 2010
}

\author{
International Academic Research Conference
}

14 - 16 April 2010

The University of Edinburgh 


\section{Abstract}

On behalf of the Steering Committee, welcome to the joint ACM/BCS 2010 "Visions of Computer Science" conference. Following the successful launch in 2008 of the conference, we again achieved a high-quality, wide-spectrum conference with a strong international profile. 


\section{Full Synopsis}

On behalf of the Steering Committee, welcome to the joint ACM/BCS 2010 "Visions of Computer Science" conference. Following the successful launch in 2008 of the conference, we again achieved a high-quality, wide-spectrum conference with a strong international profile.

The keynotes from Ross Anderson, Nicolò Cesa-Bianchi, Jon Kleinberg and Barbara Liskov, together with the accepted papers, cover a broad range of computing topics while also illuminating the socioeconomic ecosystem in which modern computing lives, and I hope we have constructed a programme that can speak to the discipline as a whole.

Continuing in the mission to energise the UK community around inspiring visions of our discipline, we chose this year to co-locate the Visions conference with the UKCRC Grand Challenges in Computing Research workshop, and I am sure that the presentations and discussions will provide valuable food for thought.

Derek McAuley, Programme Chair 


\section{Programme Committee}

\section{Programme Chairs}

Derek McAuley (Nottingham)

Simon Peyton-Jones (Microsoft)

\section{Programme Committee}

Michel Beaudouin-Lafon (LRI)

Mic Bowman (Intel)

Matthew Chalmers (Glasgow)

Fabrizio Gagliardi (Microsoft)

Carole Goble (Manchester)

Matt Grossglauser (Nokia)

David Hutchinson (Lancaster)

Jane Hillston (Edinburgh)

Marina Jirotka (Oxford)

Matt Jones (Swansea)

Ross King (Aberystwyth)

Mounia Lalmas (Glasgow)

Michael Luck (Kings College)

Julie McCann (Imperial)

Ursula Martin (Queen Mary)

Tom Melham (Oxford)

Simon Moore (Cambridge)

Colin Runciman (York) 
Nigel Shadbolt (Southampton)

Susan Stepney (York)

Alexander Wolf (Imperial)

\section{Organising Committee}

General Chair

Subramanian Ramamoorthy (Edinburgh)

Keynote Chair

Philip Wadler (Edinburgh)

\section{Steering Group}

Liz Bacon (Greenwich)

Michel Beaudouin-Lafon (I'Université de Paris-Sud)

Muffy Calder(Glasgow)

Ann DeRoeck (Open University)

Michael Fourman (Edinburgh)

Steve Furber (Manchester)

Fabrizio Gagliardi (Microsoft)

Wendy Hall (Southampton)

Dave Robertson (Edinburgh)

Alexander Wolf (Imperial) 


\section{Keynotes}

The dependability of complex socio-technical systems

Abstract: Since the invention of agriculture, we humans have been organising large systems. From armies to markets, from religions to the civil service, we have devised ways of coordinating people's efforts. But the large systems of today are turning into something qualitatively different, because of the interaction of many people with complex software and global scale: examples range from the bank payment network through Facebook to the Internet itself. Some of these systems just evolved; there are others we are trying to build deliberately, such as a future smart grid to improve energy distribution. Like it or not, our civilisation is coming to depend on all this stuff. I therefore propose a new Grand Challenge: organising what we know about the dependability of complex socio-technical systems, and figuring out how this understanding might be improved.

\section{Nicolò Cesa-Bianchi - The University of Milan}

The game-theoretic approach to machine learning and adaptation

Abstract: The development of artificial systems able to learn and adapt in a changing environment has been a central goal of Artificial Intelligence since its very beginning. From the viewpoint of the analysis of learning algorithms, which is the theme of this talk, statistical modeling has proven to be a tool of extraordinary effectiveness. However, statistics may not always be the most adequate language to describe the interaction between a learning agent and an ever-changing environment. Indeed, a parallel thread of research has emerged, where this interaction is modeled as a repeated game between agent and environment. The game-theoretic approach allows adaptation to arbitrary and even adversarial environments within a rigorous mathematical framework. This talk explores the extent to which game theory may complement or surpass statistics in the analysis of algorithms that learn and adapt.

\section{Jon Kleinberg - Cornell University}

Exploring the Structure of On-Line Social Networks: The Roles of Positive and Negative Links in Network Interaction

Abstract: Current large-scale information systems involve rich patterns of social interaction, and understanding the network structure of these interactions is crucial both for the design of the systems and for our understanding of human social dynamics at global scales. 
The social networks in many on-line settings encode a mixture of positive (friendly) and negative (antagonistic) relationships, but the bulk of research on these networks to date has focused almost exclusively on the positive interpretations of the links. We discuss how the interplay between positive and negative relationships affects the overall functioning of on-line social networks, and we connect our analysis to theories of signed networks from social psychology. In addition to providing a perspective for reasoning about the underlying networks and applications, this analysis provides one of the first largescale evaluations of these theories using on-line datasets. We find that classical theories capture certain of the underlying effects, but that they are also at odds with some of the fundamental phenomena we observe - particularly related to the evolving, directed nature of the networks.

\section{Barbara Liskov - Massachusetts Institute of Technology}

The Power of Abstraction

Abstract: Abstraction is at the center of much work in Computer Science. It encompasses finding the right interface for a system as well as finding an effective design for a system implementation. Furthermore, abstraction is the basis for program construction, allowing programs to be built in a modular fashion. This talk will discuss how the abstraction mechanisms we use today came to be, how they are supported in programming languages, and some possible areas for future research. 


\section{Papers:}

\section{Session 1: Ubiquitous Systems}

Matthew Chalmers A Population Approach to Ubicomp System Design

http://dx.doi.org/10.14236/ewic/VOCS2010.1

David Benyon, Kristina Höök and Laurence Nigay Spaces of Interaction

http://dx.doi.org/10.14236/ewic/VOCS2010.2

\section{Session 2: Theoretical Computing}

Dale Miller Finding Unity and Computational Logic http://dx.doi.org/10.14236/ewic/VOCS2010.3

Paul Cockshott, Lewis Mackenzie and Greg Michaelson Non-classical computing: feasible versus infeasible http://dx.doi.org/10.14236/ewic/VOCS2010.4

\section{Session 3: Digital Economy}

Paul Cockshott and Karen Renaud Extending Handivote to Handle Digital Economic Decisions http://dx.doi.org/10.14236/ewic/VOCS2010.5

Anil Madhavapeddy, Richard Mortier, Jon Crowcroft and Steven Hand Multiscale not Multicore: Efficient Heterogeneous Cloud Computing http://dx.doi.org/10.14236/ewic/VOCS2010.6

\section{Session 4: In the wild}

Michael Breza, Pedro Martins, Julie A. McCann, Evangelos Spyrou, Poonam Yadav and Shusen Yang Simple Solutions for the Second Decade of Wireless Sensor Networking http://dx.doi.org/10.14236/ewic/VOCS2010.7

Leysia Palen, Kenneth M. Anderson, Gloria Mark, James Martin, Douglas Sicker, Martha Palmer and Dirk 
Grunwald A Vision for Technology-Mediated Support for Public Participation \& Assistance in Mass Emergencies \& Disasters http://dx.doi.org/10.14236/ewic/VOCS2010.8 